www.nature.com/jhg

\title{
A commentary on A multiplex SNP assay for the dissection of human Y-chromosome haplogroup 0 representing the major paternal lineage in East and Southeast Asia
}

\author{
Hong Shi
}

Journal of Human Genetics (2012) 57, 5; doi:10.1038/jhg.2011.136; published online 1 December 2011

\begin{abstract}
T $\mathrm{n}$ recent two decades, human population 1 genetic studies had constructed a Y-chromosome phylogeny, ${ }^{1}$ and each haplogroup (or clade) is defined by a combination of single-nucleotide polymorphisms (SNPs). The Y-chromosome haplogroups usually have specific geographic distribution and the phylogeny of $\mathrm{Y}$ chromosome provides a powerful tool to reconstruct history of human populations and paternal pedigrees. ${ }^{1,2}$ The Y-chromosome SNPs are informative of inferring the origin, expansion and prehistoric migration of modern human populations, and they are also useful in forensic analysis. Hence, it is important to set up a rapid method identifying multiple SNPs that define one $\mathrm{Y}$ haplogroup. The multiplex SNP assay is useful for rapid genotyping. The researchers strive to develop the effective multiplex SNP assay on the human Y-chromosome typing in recent 10 years. ${ }^{3,4}$ A newly developed method was able to genotype a set of SNPs simultaneously and quickly defines the Y-chromosome haplogroup and subhaplogroups. This method
\end{abstract}

was based on a multiplexed single-base primer extension using the SNaPshot multiplex kit. ${ }^{5}$

The reported assay selected 16 Y-SNPs that define the haplogroup $\mathrm{O}$ and its subhaplogroups. As Haplogroup-O is prevalent in eastern Asia area, this assay would be highly useful in studying populations. ${ }^{5}$ The authors have optimized the reaction conditions including the SNP panel organization, primer design, and reagent concentration and so on. They have developed a relative stable protocol to analyze the Y-chromosome haplogroup $\mathrm{O}$ by multiplexing the 16 primer pairs in one single PCR reaction. The $\mathrm{T}(\mathrm{GACT})_{n}$, $\mathrm{CT}(\mathrm{GACT})_{n}, \mathrm{ACT}(\mathrm{GACT})_{n} \ldots$ tails are added into the $5^{\prime}$ extension primers so that the 16 SNPs can be separated during electrophoresis, which turned out to be a better design compared with the commonly used poly $\mathrm{T}$ tail. ${ }^{3-5}$ The only problem left for future optimization is the amplification efficiency difference among the 16 SNPs. For example, the amplifications of M175 and M324 are stronger than the other SNPs, and the signals of M7, M119 and 002611 are very weak. Overall the reported assay provides a useful tool for Y-chromosome genotyping and may apply to the other haplogroups in the future.

\footnotetext{
1 Karafet, T. M., Mendez, F. L., Meilerman, M. B., Underhill, P. A., Zegura, S. L. \& Hammer, M. F. New binary polymorphisms reshape and increase resolution of the human Y chromosomal haplogroup tree. Genome Res. 18, 830-838 (2008).

2 Underhill, P. A., Passarino, G., Lin, A. A., Shen, P., Mirazon Lahr, M., Foley, R. A. et al. The phylogeography of $Y$ chromosome binary haplotypes and the origins of modern human populations. Ann. Hum. Genet. 65, 43-62 (2001).

3 Inagaki, S., Yamamoto, Y., Doi, Y., Takata, T., Ishikawa, T., Yoshitome, K. et al. Typing of $Y$ chromosome single nucleotide polymorphisms in a Japanese population by a multiplexed single nucleotide primer extension reaction. Leg. Med. (Tokyo) 4, 202-206 (2002).

4 Yan, S., Wang, C. C., Li, H., Li, S. L. \& Jin, L. An updated tree of Y-chromosome Haplogroup $\mathrm{O}$ and revised phylogenetic positions of mutations P164 and PK4. Eur. J. Hum. Genet. 19, 1013-1015 (2011).

5 van Oven, M., van den Tempel, N. \& Kayser, M. A multiplex SNP assay for the dissection of human $Y$ chromosome haplogroup 0 representing the major paternal lineage in East and Southeast Asia. J. Hum. Genet. 57, 65-69 (2012).
} 